\title{
Eosinophilic granulomas in two dogs
}

\author{
Jae-Hoon Kim¹, Ji-Youl Jung', Sang-Chul Kang1, Young-Rak Lee ${ }^{2}$, Jin-Yong Lee, \\ Eui-Kyung Hwang ${ }^{4}$, Gye-Hyeong Woo ${ }^{5}$, Jae-Hoon Kim ${ }^{1, *}$ \\ ${ }^{I}$ College of Veterinary Medicine and Veterinary Medical Research Institute, Jeju National University, \\ Jeju 690-756, Korea \\ ${ }^{2}$ Gangnam Animal Medical Center, Busan 612-010, Korea \\ ${ }^{3}$ Cats and Dogs Animal Clinic, Seoul 121-250, Korea \\ ${ }^{4}$ College of Life Science and Natural Resources, Sangji University, Wonju 220-702, Korea \\ ${ }^{5}$ Department of Clinical Laboratory Science, Semyung University, Jecheon 390-711, Korea
}

(Accepted: November 11, 2010)

\begin{abstract}
Eosinophilic granuloma is a common hypersensitive inflammatory skin disease in cats, and rare in dogs and horses. The skin biopsies of 5 years old female Cocker spaniel and 2 years old female mixed dog had the clinical signs of skin nodules with alopecia were submitted for diagnosis. Solitary skin nodules and papillary nodules were presented on the left external ear and back of Cocker spaniel and on the external ear of mixed dog, respectively. Histopathologically, epidermis of skin showed mild to severe hyperplasia with multifocal ulceration. Small to large irregular, brightly eosinophilic foci with degenerating eosinophils and homogeneous degenerated collagens were existed in the dermis of both ear and back skin. Typical 'flame figures', a mixture of degenerated collagen and degranulated eosinophils, were observed in both cases. Based on the histopathologic findings and special staining characters, 2 cases were diagnosed as canine eosinophilic granuloma. This is the first report for the eosinophilic granuloma of dogs in Korea.
\end{abstract}

Keywords : dog, eosinophilic granuloma, flame figure, nodule, skin

\section{Introduction}

Eosinophilic granuloma complex (EGC) is an inflammatory skin disease that has been reported in cats, dogs, and horses $[2,6,7]$. EGC is not a specific disease but simply several cutaneous reactions, and most commonly seen in cats. Feline eosinophilic granulomas (EG also called linear granuloma and collagenolytic granuloma) occur on the caudal thighs, face, oral cavity, and occasionally on the footpad $[2,6]$. Cutaneous lesions are typically well-circumscribed, raised, firm, alopecic, erythematous plaques with a characteristic linear configuration [2].

Canine EG is characterized by lesions resembling feline EG $[2,7]$. They are most common in the oral cavity and less frequent in haired skin such as inner thighs, ventral abdomen, flanks, and prepuce [2, 7]. This disease most commonly presents as single to multiple ulcerated lesions, in oral cavity, often on the lateral or ventral surfaces of the tongue or on the soft palate [2, $3,8]$. The cutaneous form is characterized by multiple papules, nodules, and plaques. Oral EG occur almost exclusively in male Siberian huskies and male Cavalier King Charles spaniels, whereas cutaneous lesions have no recognized breed predilection [3, 7, 8]. Canine EG probably are of multifactorial allergic etiology. Both clinical and histopathologic features suggest a hypersensitivity reaction.

In this paper, we report two cases of EG in female dogs. To the author's knowledge, this is the first case reported in Korea.

\section{Cases}

The skin biopsies of 5 years old female Cocker spaniel (Case 1) and 2 years old female mixed dog (Case 2)

*Corresponding author

Tel: +82-64-754-3387, Fax: +82-64-702-9920

E-mail: kimjhoon@jejunu.ac.kr 


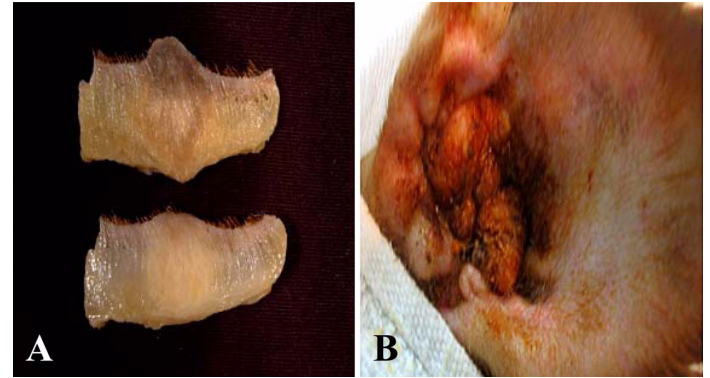

Fig. 1. Dog, Skin nodules. Note well-circumscribed, solitary, raised nodule in back skin of case 1 (A) and papillary mass in the external ear canal of case 2 (B).

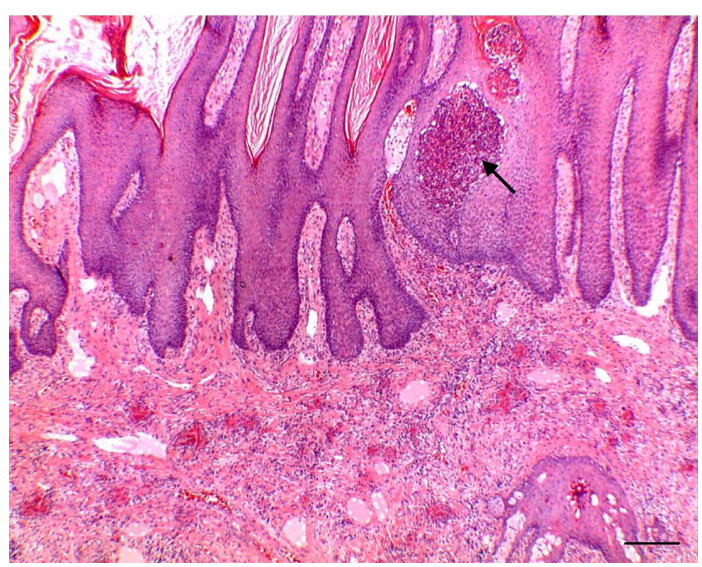

Fig. 2. Papillomatous lesion from external ear canal of case 2. Epidermis showed severe hyperplasia with rete ridge down growth and eosinophilic pustule (arrow). H\&E, Scale bar $=200 \mu \mathrm{m}$.

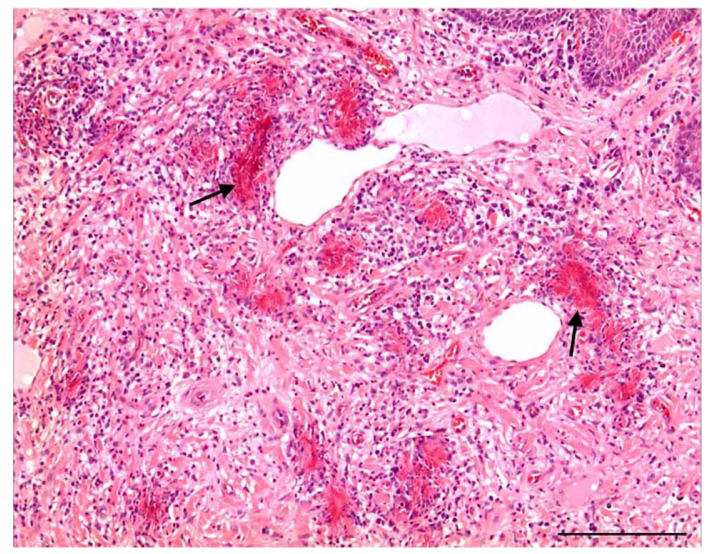

Fig. 3. Multifocal foci of densely eosinophilic collagens surrounded by eosinophils (flame figures, arrows) in dermis. H\&E, Scale bar $=200 \mu \mathrm{m}$. were submitted to the Veterinary Pathology Laboratory of Jeju National University because of skin nodules with alopecia. Solitary skin nodules and papillary nodules were presented on the left external ear and back of Cocker spaniel and on the external ear of mixed dog, respectively. Tissues biopsy samples were fixed in $10 \%$ neutral buffered formalin, processed in a routine method, and stained with hematoxylin and eosin (H\&E) for light microscopic examination. Special staining such as Masson's trichrome and Luna's staining also performed on paraffin embedded tissue sections.

Grossly, the nodule of the back skin was solitary, round to oval shape, raised, alopecic, and firm. On cut surface, the dermal nodule was well-circumscribed with normal cutaneous tissues (Fig. 1A). And raised, papillary masses were occupied in the external ear canal (Fig. 1B).

Histopathologically, epidermis of skin showed severe papillary hyperplasia with prominent rete ridges down growth (Fig. 2). Keratinized squamous epithelium was covered with dense crust, and multifocal intercellular edema and spongiosis were seen. Multifocal ulceration was noted in case 1. Small pustules composed of eosinophils were sometimes occupied in epidermis. Many necrotic areas were presented in the center of dermal lesion. Small to large irregular, brightly eosinophilic foci with degenerating eosinophils and homogeneous degenerated collagens were existed in the dermis both ear and back skin (Fig. 3). Typical 'flame figures' had many foci of collagen degeneration with peripheral, small, radiating projections surrounded by eosinophils, lymphocytes, macrophage, and the degenerated inflammatory cells (Fig. 4A). According to Masson's trichrome method, some collagen fibers within flame figures spaces appeared normal, while others appeared to be partially or wholly degenerate (normal collagen-blue, degenerated collagen-red) (Fig. 4B). Collagen fibers were coated by degranulating eosinophils using Luna's staining (Fig. 4C). Many eosinophils and mononuclear cells also infiltrated around blood vessels and adnexa structures.

\section{Discussion}

Based on the gross findings, histopathologic features, and special staining methods, these cases were diagnosed as EG in dogs. The EGC in the cat actually consists of three controversially similar diseases [2]. Classically, three lesions such as the indolent ulcer, the eosinophilic 


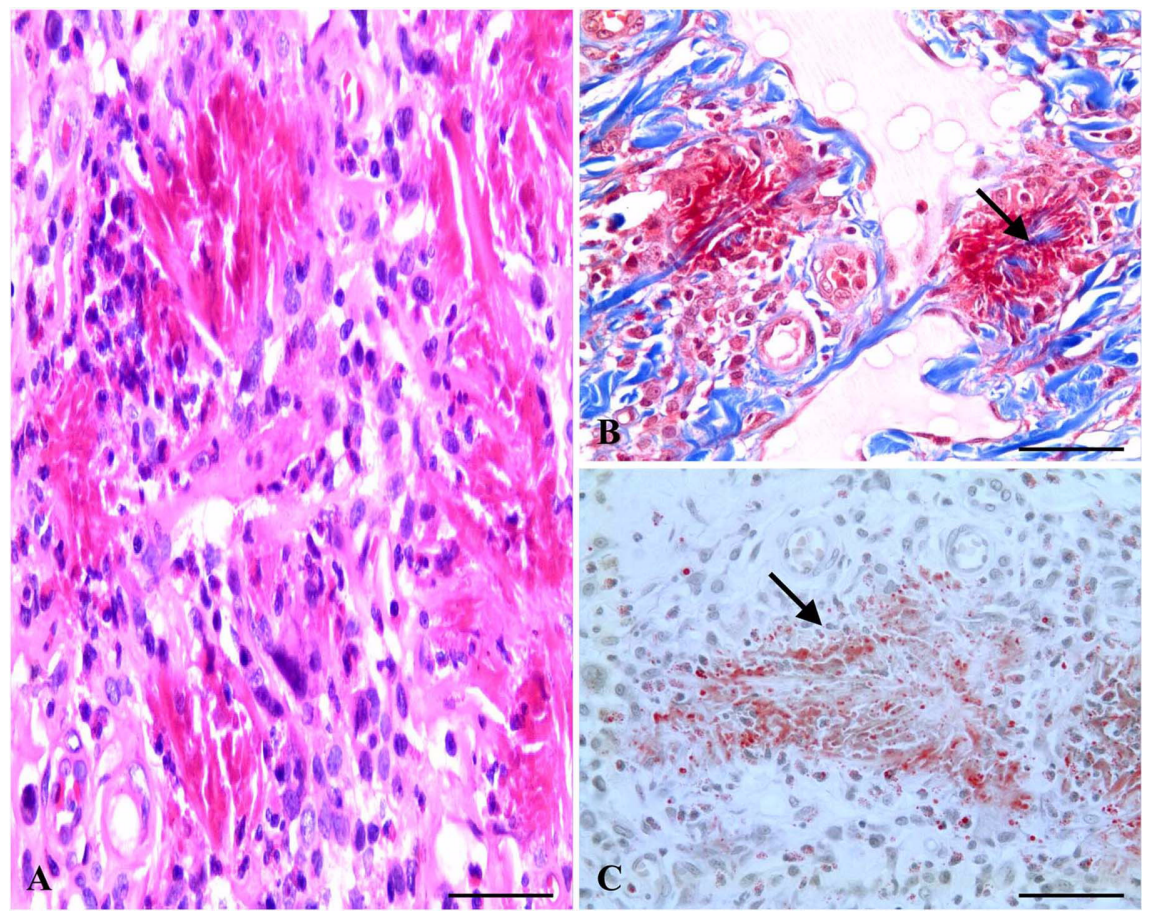

Fig. 4. Degranulating eosinophils are accumulated along collagen fibers (A). H\&E. Some blue collagen fibers have segmental red staining abnormalities (B, arrow). Masson's trichrome. Note degranulation from eosinophils (C, arrow). Luna's staining. Scale bars $=50 \mu \mathrm{m}$.

plaque, and the EG have been characterized in feline EGC. These diseases are best thought of as inflammatory reactions of the skin and may be associated with hypersensitivity diseases $[2,7]$. The cause of EGC is unknown, however food allergy, atopy, and insect allergy often has been associated with these lesions. Since antibiotic therapy clears or significantly improves some lesions, bacterial infection also may be implicated. Because three clinical variant of EGC have common histopathologial features, it has been suggested that the use terms eosinophilc plaque, indolent ulcer, and eosinophilic granuloma be restricted to clinical dermatology, while the term eosinophilic dermatoses be used in diagnostic dermatopathology [6].

EG has been reported in the dog as a rare lesion of the skin and oral cavity [3, 8]. Multiple cutaneous nodules and raised plaques on the ventral abdomen, prepuce, flanks, and limbs have been reported. Individual case involved in the eyelid of a Siberian husky and in the nasal skin in a 12-year-old dog was previously reported $[4,10]$. Unusual location of focal proliferative EG in the external ear canal of four dogs was described in USA [9]. This report describes EG in female Cocker spaniel and female mixed dog, a breed in which the disease has been rarely diagnosed. In addition, our two cases were found in external ear and back, unusual sites of EG occurrence. The histopathologic lesions in these two dogs were resembled that seen in Siberian huskies and Cavalier King Charles spaniels with EG.

Feline EGC is characterized histopathologically by an intense dermal eosinophilic infiltrate accompanied by deposition of amorphous to granular debris that appears eosinophilic or basophilic on H\&E staining [6]. These characteristic deposits have been termed "flame figures", and these have been reported to comprise a mixture of degenerated collagen fibers and degranulated eosinophils [7]. However, on trichrome-stained histopathologic sections, normally stained collagen fibers have been identified in the center of both flame figures and large foci of collagen degeneration [5]. Therefore eosinophilic degranulation around normal collagen bundles seemed to represent the major pathogenetic event in EGC [6]. According to electron microscopic study of feline EGC lesions, flame figures are constituted by eosinophils, 
undergoing both cytolysis and piecemeal degranulation, around partially disrupted but undamaged collagen fibrils [1]. In dogs, the lesions reported as EG histologically resemble the EG of cats.

For the differential diagnosis of EG, infectious ulcer or granulomas, trauma, and several tumors such as squamous cell carcinoma, mast cell tumor, lymphoma, fibrosarcoma, and mammary carcinoma should be considered [7]. Skin scraping and cytologic examination are recommended to exclude infectious diseases and neoplasms [2]. However definitive diagnosis of the EG complex must be made on histopathology.

The EG in most cases responds to immunosuppressive therapy $[2-4,10]$. The dog with this disease has been treated with per-oral administration of prednisone or prednisolone at $1 \mathrm{mg} / \mathrm{kg} /$ day for one week, then tapering down over the course of four to six weeks. Systemic antibiotics should also be tried initially in the case of eroded or ulcerated lesions.

\section{Conclusion}

The clinicopathologic features of these cases are consistent with EG, and the first report of this disease in Cocker spaniel. Hence EG should take into consideration in the differential diagnosis of erythematous plaques or nodules in oral cavity and ear skin of young dogs, regardless of breed. Although canine EG is clinically distinctive, histopathology is very significant and helpful in establishing the definitive diagnosis.

\section{References}

1. Bardagí M, Fondati A, Fondevila D, Ferrer L.
Ultrastructural study of cutaneous lesions in feline eosinophilic granuloma complex. Vet Dermatol 2003, 14, 297-303.

2. Bloom PB. Canine and feline eosinophilic skin diseases. Vet Clin North Am Small Anim Pract 2006, 36, 141-160.

3. Bredal WP, Gunnes G, Vollset I, Ulstein TL. Oral eosinophilic granuloma in three cavalier King Charles spaniels. J Small Anim Pract 1996, 37, 499-504.

4. da Silva Curiel JM, Kraus KH, Brown TP, Chastain CB. Eosinophilic granuloma of the nasal skin in a dog. J Am Vet Med Assoc 1988, 193, 566-567.

5. Fernandez CJ, Scott DW, Erb HN. Staining abnormalities of dermal collagen in eosinophil- or neutrophil-rich inflammatory dermatoses of horses and cats as demonstrated with Masson's trichrome stain. Vet Dermatol 2000, 11, 43-48.

6. Fondati A, Fondevila D, Ferrer L. Histopathological study of feline eosinophilic dermatoses. Vet Dermatol 2001, 12, 333-338.

7. Gross TL, Ihrke PJ, Walder EJ, Affolter VK. Skin Diseases of the Dog and Cat. 2nd ed. pp. 355-362, Blackwell Science, Oxford, 2005.

8. Madewell BR, Stannard AA, Pulley LT, Nelson VG. Oral eosinophilic granuloma in Siberian husky dogs. J Am Vet Med Assoc 1980, 177, 701-703.

9. Poulet FM, Valentine BA, Scott DW. Focal proliferative eosinophilic dermatitis of the external ear canal in four dogs. Vet Pathol 1991, 28, 171-173.

10. Vercelli A, Cornegliani L, Portigliotti L. Eyelid eosinophilic granuloma in a Siberian husky. J Small Anim Pract 2005, 46, 31-33. 